\title{
Lymphoid follicles in (very) severe COPD: beneficial or harmful?
}

\author{
G.G. Brusselle*, T. Demoor*, K.R. Bracke*, C-A. Brandsma* and W. Timens ${ }^{\#}$
}

ABSTRACT: Inflammation is a main pathogenetic factor in the development and progression of chronic obstructive pulmonary disease (COPD). Recently, it has become clear that not only the innate, but also the specific immune response plays a role. A striking finding, in particular in lungs of patients with severe COPD, often with a predominant emphysema phenotype, is the presence of B-cell follicles. As seen in other tissues, these follicles are the result of lymphoid neogenesis. The finding of oligoclonality in B-cell follicles in COPD suggests that they play a role in local antigen specific immune responses. To date, it is not known which antigens may be involved; microbial antigens, cigarette smoke-derived antigens and antigens from extracellular matrix breakdown products have been suggested. Consequently, the pathogenetic role of this follicular $B$-cell response is not yet clear. It might be protective against microbial colonisation and infection of the lower respiratory tract and, therefore, beneficial, or it could be of a more harmful (autoimmune) nature, directed against lung tissue components. It is necessary to determine the specific antigen(s) and to explore the exact role of the COPD related B-cell response in order to include modulation of this response and develop therapeutic options.

KEYWORDS: Chronic obstructive pulmonary disease, immune response, inflammatory response, lymphocytes, review

hronic obstructive pulmonary disease (COPD) is a chronic inflammatory disorder in different compartments of the lung, including a mixture of small airway disease (obstructive bronchiolitis) and parenchymal destruction (emphysema). COPD is associated with a relentlessly progressive course and increasing morbidity, disability and mortality worldwide [1]. Smoking is known to be the major aetiological factor, yet the exact pathogenetic mechanisms have not yet been elucidated. Smoking cessation is the only effective treatment, but it only partially attenuates further loss of lung function. In those COPD patients who have fully ceased smoking, pulmonary inflammation persists [2-4]. The mechanism that propagates this inflammation is incompletely understood.

Since COPD is characterised by chronic airflow limitation that is not fully reversible and usually progressive, the Global Initiative for Chronic Obstructive Lung Disease (GOLD) has introduced a five-stage classification for the severity of COPD based on measurements of airflow limitation during forced expiration, as measured by forced expiratory volume in $1 \mathrm{~s}$ [5]. The progression of
COPD is associated with infiltration of the wall of small airways by innate and adaptive inflammatory immune cells. The inflammatory cells that were initially identified as being the most important in the pathogenesis of COPD were neutrophilic granulocytes and macrophages, which both belong to the innate immune system. The importance of neutrophils and macrophages has been confirmed by animal studies showing the necessity of both these cells for the induction of experimental emphysema by cigarette smoke (CS) $[6,7]$. It later became clear that the specific immune system also plays a role; in particular CD8 positive T-lymphocytes were associated with smoking and subsequent risk for COPD [8, 9]. Recently, B-lymphocytes organised into peribronchiolar lymphoid follicles have been associated with severe COPD [10] and, in addition, in COPD such follicles were also found in the parenchyma [11], whereas B-cells were increased in large airways [12]. As the antigen inducing this follicular B-cell response is as yet unknown, it has yet to be decided whether and to what extent this response is of beneficial or harmful nature. This review aims to shed light on the nature of this response, the possible aetiological factors and to

\section{AFFILIATIONS}

*Dept of Respiratory Medicine, Ghent University Hospital, Ghent, Belgium, and

\#Dept of Pathology, University Medical Center Groningen, Groningen, The Netherlands.

CORRESPONDENCE

G.G. Brusselle

Dept of Respiratory Medicine Ghent University Hospital De Pintelaan 185

9000 Ghent

Belgium

E-mail: guy.brusselle@ugent.be

Received:

Oct 032008

Accepted after revision: Jan 172009 
present our views on the possible role in the pathogenesis and progression of COPD.

\section{LYMPHOID FOLLICLES IN COPD}

Whereas one of the first reports on the presence of B-cell follicles in COPD dates from 1992 [13], quite recently attention has been redrawn to a possible role of B-cells. An increase of small airways containing B-cell follicles was found in patients with severe COPD compared to normal subjects and patients with mild-to-moderate COPD. Furthermore, the progression of COPD from GOLD stage 0 to GOLD stage 4 was indeed clearly associated with the number of small airways containing these lymphoid follicles $[10,14]$. Furthermore, the presence of B-cell follicles was demonstrated not only in relation to small airways, but also in the lung parenchyma of COPD patients (fig. 1) and mice chronically exposed to CS [11]. An increase of individual B-cells in COPD was also observed, in particular in bronchial biopsy studies of large airways and, in addition, nonfollicular B-cell aggregates were identified [12, 15].

It is important to note that the original publication demonstrating lymphoid follicles in patients with severe and very severe COPD [10] could encompass a selection bias, since all lung specimens of GOLD stage 3 and 4 COPD were obtained from lung volume reduction surgery specimens of patients with severe emphysema enrolled in the National Emphysema Treatment Trial (NETT). However, in the study of VAN DER STRATE et al. [11], the majority of the human lung tissue samples (six out of eight patients with very severe COPD) were obtained at lung transplantation (explant lungs). Nevertheless, more studies are needed to assess the role of lymphoid follicles in different COPD disease stages and phenotypes, including severe COPD with predominant bronchiolitis.

With respect to B-cell function and development, a distinction should be made between B-cell aggregates without a specific functional architecture and B-cells organised in (primary or secondary) follicles; this is also reflected in their supposed functional role. Lymphoid aggregates consist of mature, memory B-cells and/or T-cells without functional organisation, thus mainly available for local priming and activation. The functional organisation (see below) of primary and secondary lymphoid follicles (the latter containing germinal centres) consists of a specific arrangement of memory and naïve B-cells, T-cells, dendritic cells (DCs) and follicular dendritic cells (FDC), which allows for B- and T-cell priming, clonal expansion, antigen retention (mostly as immune complexes), somatic hypermutation, affinity maturation and immunoglobulin (Ig) class switching [16-18]. However, it should be kept in mind that over time the distinction is not always clear. When local circumstances and factors allow, lymphoid follicles can develop out of any lymphoid aggregate. Importantly, only the micro-anatomic organisation of a lymphoid follicle, with the ability of germinal centre development, allows for its specific functions [16-18].

In COPD, the follicles in the lung $[10,11,14]$ consist of large aggregates of B-cells that are surrounded by lower numbers of mainly CD4 (80-90\%) and some CD8-positive T-cells. These aggregates can be considered lymphoid follicles, since FDC were present in these aggregates, expressing CD21 and CD35 (fig. 1). The presence of FDC, involved in affinity maturation and isotype switching of B-cells, suggests antigen-driven proliferation. The B-cells were mainly IgM-positive and IgDnegative, which suggests that these B-cells may have been activated to some extent. Moreover, a predominant part of the infiltrate appeared to be CD27-positive, a marker for memory B-cells [19]. Interestingly, CD138-positive (plasma) cells were detectable in the direct vicinity of these follicles. Furthermore, CD40 and CD40 ligand, important co-stimulatory molecules, were expressed in these follicles. The Ki-67 antigen was detected on small central clusters of B-cells in the follicles, suggesting an early germinal centre reaction [17].

Most importantly, B-cells isolated from the B-cell follicles have been demonstrated to be oligoclonal in nature [11], suggesting antigen-specific induction of the B-cell follicles. In this study, 10 lymphoid follicles were isolated by laser microdissection from the lung tissue of eight patients with COPD [11]. Sequence analysis of the Ig rearrangements revealed the presence of 12 different B-cell contigs with one or more sequence variation in 10 of the contigs. In seven out of the eight patients, clonal B-cell populations were observed, whereas in one patient only unrelated sequences were observed. The seven patients with related Ig sequences demonstrated the presence of ongoing mutations. The latter indicates oligoclonal B-cell proliferation in response to stimulation with as yet unknown antigen(s).

With respect to B-cell follicles in the lung, some authors regarded these as part of the mucosal immune system and thus as so-called bronchus associated lymphoid tissue. However, in most normal lungs no lymphoid tissue is observed at all [20]. Lymphoid follicles have been observed in the human lung in (subclinical or clinical) lung disease, either associated with airways or with parenchyma. Consequently it seems most logical to regard this as so-called lymphoid neogenesis and, thus, as an ectopic lymphoid tissue $[16,21]$. Such lymphoid tissues are also called tertiary lymphoid organs (TLOs), as opposed to primary (bone marrow and thymus) and secondary lymphoid organs (lymph nodes, Peyer's patches and spleen).

The term lymphoid neogenesis refers to the development of organised lymphoid structures which resemble secondary lymphoid organs in tissues that are targeted by chronic inflammatory processes, such as infection and autoimmunity $[16,22]$. The purpose of immune responses is primarily the eradication of pathogens. However, certain antigens are difficult to eradicate and result in sustained immune responses leading to chronic inflammation. In tissues that harbour the target antigens of chronic adaptive immune responses, infiltration occurs by macrophages, DCs, T-cells, B-cells and plasma cells. These cells frequently organise themselves at an anatomic and functional level as in secondary lymphoid organs, leading to the formation of B-cell follicles and T-cell areas [23]. This lymphoid neogenesis, also called TLO formation, is a dynamic process starting with sparse lymphocytic infiltrates that evolve into aggregates and eventually organise in B-cell follicles with germinal centres and distinct Tcell areas containing DCs and high endothelial venules (fig. 2). High endothelial venules regulate the extravasation of naïve Band T-cells. Thus, there are remarkable similarities between the structure of secondary lymphoid organs and TLOs, including the compartmentalisation in distinct B-cell areas (follicles) and 

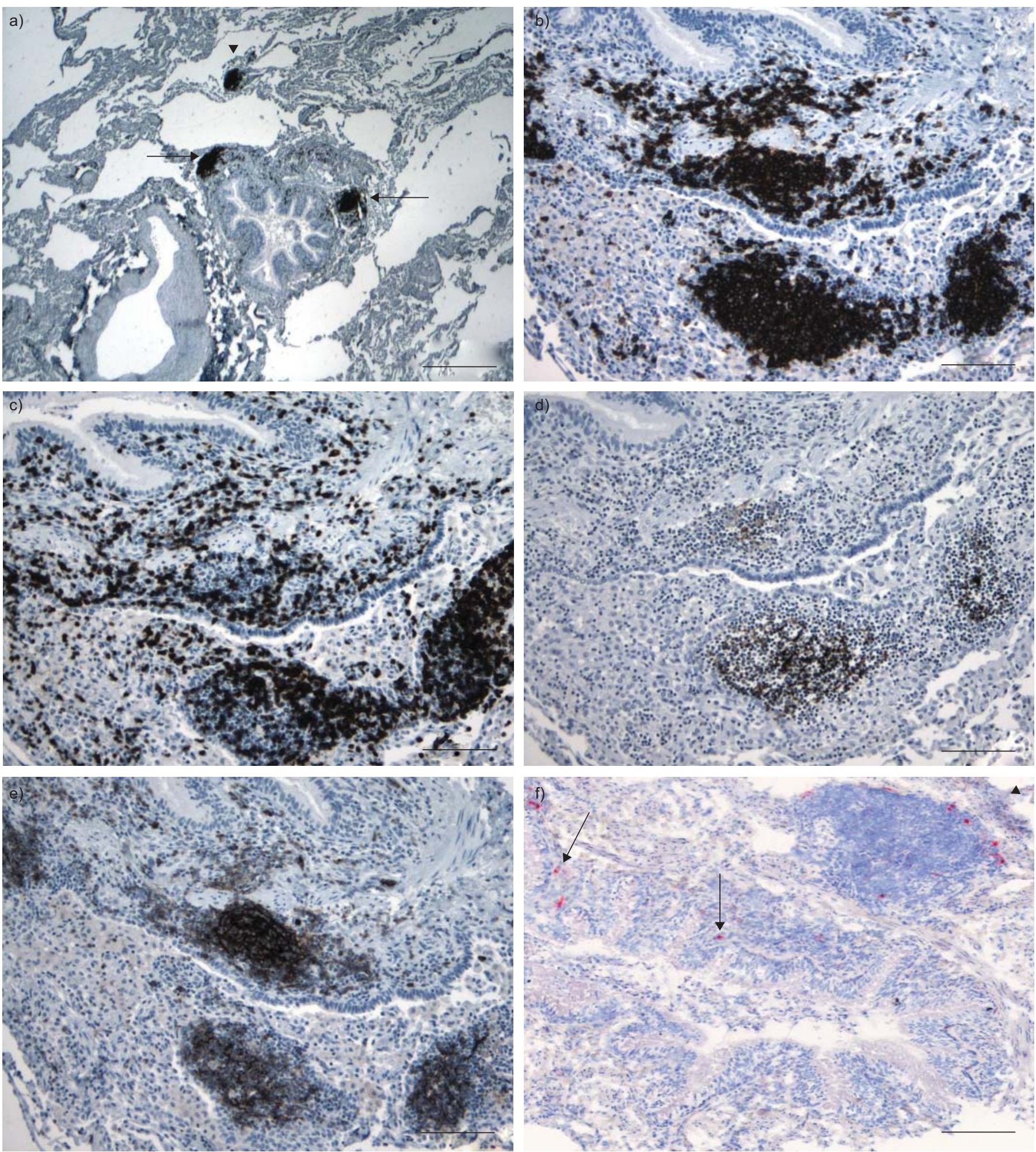

FIGURE 1. Lymphoid follicles in chronic obstructive pulmonary disease. a) CD20: overview of emphysematous lung tissue with B-cell follicles apposing a small airway (arrows) and a small B-cell aggregate in the parenchyma (arrowhead). b) CD20 staining of all B-cells. c) CD3 staining of apposing T-cells. d) CD21 (complement C3dreceptor) showing delicate staining of follicular dendritic cells. e) CD35 (complement C3b-receptor) showing more intense staining of follicular dendritic cells and weak staining of B-cells. f) CD83 staining of mature myeloid dendritic cells, showing subepithelial location (arrows), as well as presence in the rim (T-cell zone) of a lymphoid follicle (arrowhead). a) Scale bars $=1,000 \mu \mathrm{m}, \mathrm{b}-\mathrm{f}$ ) scale bars $=200 \mu \mathrm{m}$. 

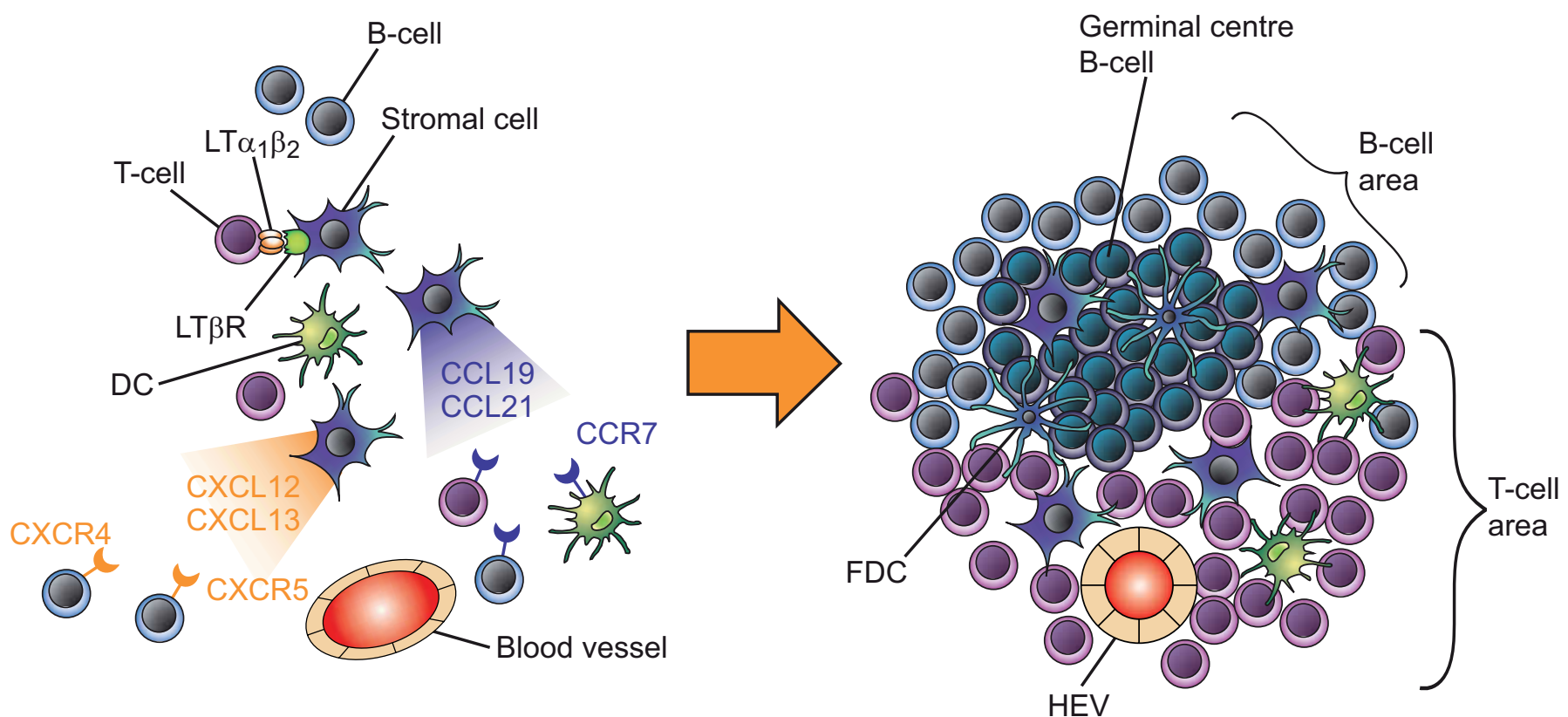

FIGURE 2. Lymphoid neogenesis in chronic inflammation. a) In chronic inflammation, activated lymphocytes expressing lymphotoxin- $\alpha-\beta$ heterotrimer (LT $\left.\alpha_{1} \beta_{2}\right)$ interact with the lymphotoxin $(\mathrm{LT}) \beta$ receptor on neighbouring stromal cells. The stromal cell stimulation induces the expression of lymphoid chemokines (CC-chemokine ligands (CCL)19, CCL21, CXC-chemokine ligands (CXCL)12 and CXCL13) along with adhesion molecules allowing the recruitment of naïve lymphocytes and dendritic cells (DCs). b) Prolonged inflammatory stimulation will lead to the organisation of lymphoid aggregates into lymphoid follicles. Germinal centres may form within the B-cell zone with highly specialised follicular dendritic cells (FDC). High endothelial venules (HEV) expressing lymphoid chemokines and adhesion molecules ensure lymphocyte and DC recruitment.

T-cell areas (paracortex). However, in contrast to lymph nodes, TLOs are not supplied by afferent lymph vessels and are not encapsulated.

\section{LYMPHOID NEOGENESIS IN MURINE MODELS OF COPD}

In vivo animal models can help to unravel the cellular and molecular mechanisms underlying the pathogenesis of COPD in general and of lymphoid neogenesis in particular [24-26]. Mice represent the most favoured animal species with regard to the study of the immunological mechanisms of diseases, as several hundreds of inbred strains and specialised stocks (e.g. mutants) are available and since they offer the opportunity to manipulate gene expression (e.g. transgenic and knock-out mice). Lymphoid follicles have been described not only in CSbased murine models of COPD, but also in pathogen-based models of COPD and in transgenic mice over-expressing pro-inflammatory cytokines in the bronchial or alveolar epithelium.

\section{CS-induced lymphoid follicles}

When the time-course of CS-induced pulmonary inflammation is determined in wild-type mice, a progressive accumulation of innate inflammatory cells (i.e. neutrophils, macrophages and DCs) in the airways and lung parenchyma is demonstrated from day 3 onwards. In addition to the persistent accumulation of innate inflammatory cells upon prolonged exposure to CS, a clear and progressive infiltration of adaptive immune cells (i.e. B-cells and T lymphocytes, both CD4+ and CD8+ T-cells) has been observed in bronchoalveolar lavage fluid and lungs of mice exposed to CS for 3-6 months [11, 27]. Inflammatory infiltrates, with a predominantly lymphoid character, were found to be present around bronchioles and in the lung parenchyma of mice after chronic CS exposure, similar to the lymphoid follicles detected in lung sections of patients with COPD. Lymphoid follicles consisting of B-cells and FDC with adjacent T-cells were detected in mice that had developed pulmonary inflammation after smoking for 6 months [11]. The increase in the number and the size of B-cell follicles was progressive with time and correlated with the (progressive) enlargement of alveolar airspaces, as evidenced by an increase in mean linear intercept. The development of emphysema in CS-exposed mice has been shown to be strain dependent. Although lymphoid follicles have been described in several strains of CS-exposed mice (e.g. BALB/c, C57BL/6 and C3H) [27-29], a formal head-to-head comparison of lymphoid neogenesis in different murine strains has not yet been performed. The number and size of peribronchial lymphoid follicles was significantly attenuated in chronically CS-exposed chemokine receptor CCR5 and CCR6 knock-out mice compared with wild-type animals, indicating a prominent role of chemokines in lymphoid neogenesis $[28,30]$.

\section{Pathogen and lipopolysaccharide-induced lymphoid follicles}

Several bacteria, including nontypeable Haemophilus influenzae (NTHi), commonly colonise the lower airways of patients with (severe) COPD. Exposure of mice to an aerosolised NTHi lysate every 8 weeks induced infiltration of macrophages, CD8+ T-cells and B-cells around airways and blood vessels, including rounded peribronchial lymphoid aggregates [31]. Lipopolysaccharide (LPS) is a major pro-inflammatory glycolipid component of the bacterial cell wall of Gram-negative bacteria (such as NTHi). Interestingly, repeated intratracheal instillation of LPS in mice resulted in persistent chronic 
pulmonary inflammation, characterised by peribronchial and perivascular lymphocytic aggregates (consisting of CD4+ and CD8+ T lymphocytes and CD19+ B lymphocytes) [32]. These in vivo studies demonstrate that some pathological changes of COPD, especially lymphoid aggregates, can be mimicked in mice after repeated exposure to either Gram-negative bacterial lysates or a component of the Gram-negative bacterial cell wall, i.e. LPS. Finally, MOYRON-QUiROz et al. [33] demonstrated that an infectious challenge with influenza virus in mice can trigger lymphoid chemokine expression and lymphoid neogenesis in the lungs, even in lymphotoxin (LT) $\alpha$-deficient mice that lack secondary lymphoid organs [33].

\section{Spontaneous development of lymphoid aggregates in lungs of transgenic mice}

Transgenic mice were generated in which human interleukin (IL) $-1 \beta$ is conditionally (with a doxycycline-inducible system) and specifically (by the Clara cell secretory protein promoter) expressed in the lung epithelium, especially in the bronchioli [34]. Induction of IL-1 $\beta$ expression in the lungs of adult mice caused pulmonary inflammation, distal airspace enlargement, airway wall fibrosis and lymphocytic aggregates in the airways. Human IL-6 and IL-6 receptor (IL-6R) double transgenic mice also developed mononuclear cell accumulation in peribronchovascular regions and lymphoid tissue-like structures expressing the chemokine CXCL13, which is indispensable for lymphoid organogenesis [35]. In separate studies, transgenic mice over expressing tumour-necrosisfactor (TNF)- $\alpha$ in the lung under the surfactant protein $C$ (SPC) promoter have been shown to produce elements of both emphysema and pulmonary fibrosis [36, 37]. Detailed histological analysis of SPC/TNF- $\alpha$ transgenic mice showed major heterogeneous abnormalities of the lung parenchyma, including infiltration of macrophages and lymphocytes into the lungs, which was most prominent in the subpleural regions, as well as distortion of alveolar structures due to septal thickening [38]. Since the pro-inflammatory cytokines IL-1 $\beta$, IL-6 and TNF- $\alpha$ have pleiotropic effects and have been implicated in multiple inflammatory disorders, it is clear that the observed pathological abnormalities in these transgenic mice can be involved in several human diseases, including not only COPD/emphysema but also lymphocytic fibrosing alveolitis, leading to pulmonary fibrosis.

\section{LYMPHOID NEOGENESIS IN OTHER DISEASES}

Lymphoid follicles are observed in various lung diseases other than COPD. Often, a long-term fibrosing process has been going on with, in due time, development of lymphoid aggregates and lymphoid follicles. This is observed in usual interstitial pneumonia/end-stage pulmonary fibrosis and also in nonspecific interstitial pneumonia and bronchiectasis. In bronchiectasis, follicles are not only observed in the airway wall but also in the surrounding parenchyma, which often shows varying fibrosis and chronic inflammation. In most of the above conditions, stasis of cellular debris and mucus is present in distorted airspaces, in which all kinds of microorganisms are present, often with clinical or subclinical infections. Therefore, an antimicrobial origin of the specific immune response giving rise to the follicles is quite likely in these diseases, although other origins cannot be excluded.
Lymphoid interstitial pneumonia (LIP) is an interstitial lung disease characterised by an extensive proliferation of small mature B- and T-cells; in general, mainly in the alveolar interstitium. However, a form with follicular bronchiolitis is often also considered to be part of this spectrum. Development of lymphoid follicles, most with florid germinal centres, is a general feature of LIP. At present LIP is considered a pulmonary manifestation related to different autoimmune diseases; auto-antigens are also most likely underlying the immune response giving rise to the lymphoid follicles. With respect to the airways, follicles are seen in bronchiectasis as described above, but the most prominent airway manifestation of lymphoid follicles is in follicular bronchiolitis. This disease condition is quite rare, sometimes related to autoimmune disease, and often considered as part of LIP (see above). As a pathological diagnosis it has been observed in association with immunodeficiency, such as in HIV, but also as an idiopathic disease.

Lymphoid neogenesis has also been described outside the lungs in several chronically inflamed tissues from patients with different underlying diseases, mainly autoimmune and infectious diseases. Human chronic inflammatory diseases with lymphoid neogenesis encompass rheumatoid arthritis, Graves' disease (hyperthyroidism), Hashimoto's thyroiditis (hypothyroidism), myasthenia gravis, multiple sclerosis, Crohn's disease, ulcerative colitis and chronic hepatitis C. The respective target tissues of the TLO formation are diverse and include the joints, the thyroid gland, the thymus, the central nervous system, the gut and the liver. In several autoimmune diseases, the self-antigen, recognised by antibodies generated in ectopic germinal centres, has been detected in lymphoid follicles (e.g. thyroglobulin and thyroperoxidase in Hashimoto's thyroiditis) [16].

\section{CELLULAR AND MOLECULAR MECHANISMS OF LYMPHOID (NEO)GENESIS}

\section{Similarities in development of secondary lymphoid organs} versus TLOs

The organisation of lymph nodes during organogenesis is the result of a highly coordinated interplay between haematopoietic cells, mesenchymal stromal cells, adhesion molecules, cytokines and chemokines (fig. 3). The key players are members of the TNF family, mainly $\mathrm{LT} \alpha_{1} \beta_{2}$ and its receptor $\operatorname{LT} \beta R[39,40]$. Binding of membrane-bound $\mathrm{LT} \alpha_{1} \beta_{2}$, expressed by haematopoietic inducer cells to LT $\beta R$ on the surface of stromal organiser cells, induces the expression of adhesion molecules, including intercellular adhesion molecule-1, vascular cell-adhesion molecule-1, mucosal addressin cell-adhesion molecule-1 and peripheral node addressin. The interaction between haematopoietic inducer cells and stromal cells via the $\mathrm{LT} \alpha_{1} \beta_{2}$-LT $\beta \mathrm{R}$ pathway also induces the expression of homeostatic lymphoid chemokines, such as CC-chemokine ligand (CCL)19 and CCL21, as well as CXC-chemokine ligand (CXCL)12 and CXCL13 [41]. These lymphoid chemokines orchestrate the lymphocyte homing and compartmentalisation of the lymphoid organs. CCL19 and CCL21 are produced by stromal cells and regulate the homing of CCR7 $7^{+}$naive T-cells and mature DCs to T-cell areas. CXCL12 and CXCL13 are produced by FDC, thought to originate from mesenchymal stromal cells, and attract CXCR5+ B-cells into the follicles. 
Activated tissue fibroblasts (e.g. fibroblast-like synoviocytes) share several features with the stromal cells that form the reticular network of secondary lymphoid organs, including the production of lymphoid chemokines (such as CXCL12, formerly called stromal cell-derived factor 1).

\section{DCs}

In the respiratory tract, myeloid DCs form a dense network of antigen-presenting cells in or just beneath the epithelium and survey the airways for exogenous and endogenous danger signals [42]. Recently, a significant increase in Langerin+ DCs has been demonstrated in the small airways of patients with COPD compared with healthy smokers and never-smokers [43]. As in the gastrointestinal tract, the accumulation of immature Langerin+ DCs was associated with increased CCL20 levels in induced sputum and lung tissue of COPD patients [43]. Upon antigen uptake and activation, DCs lose all chemokine receptors except CCR7, which regulates the migration of DCs towards the regional lymph nodes as well as the influx of naïve T-cells into the paracortex. It is conceivable that similar mechanisms also direct the migration of DCs and T-cells towards the T-cell zone of lymphoid follicles (fig. 3).

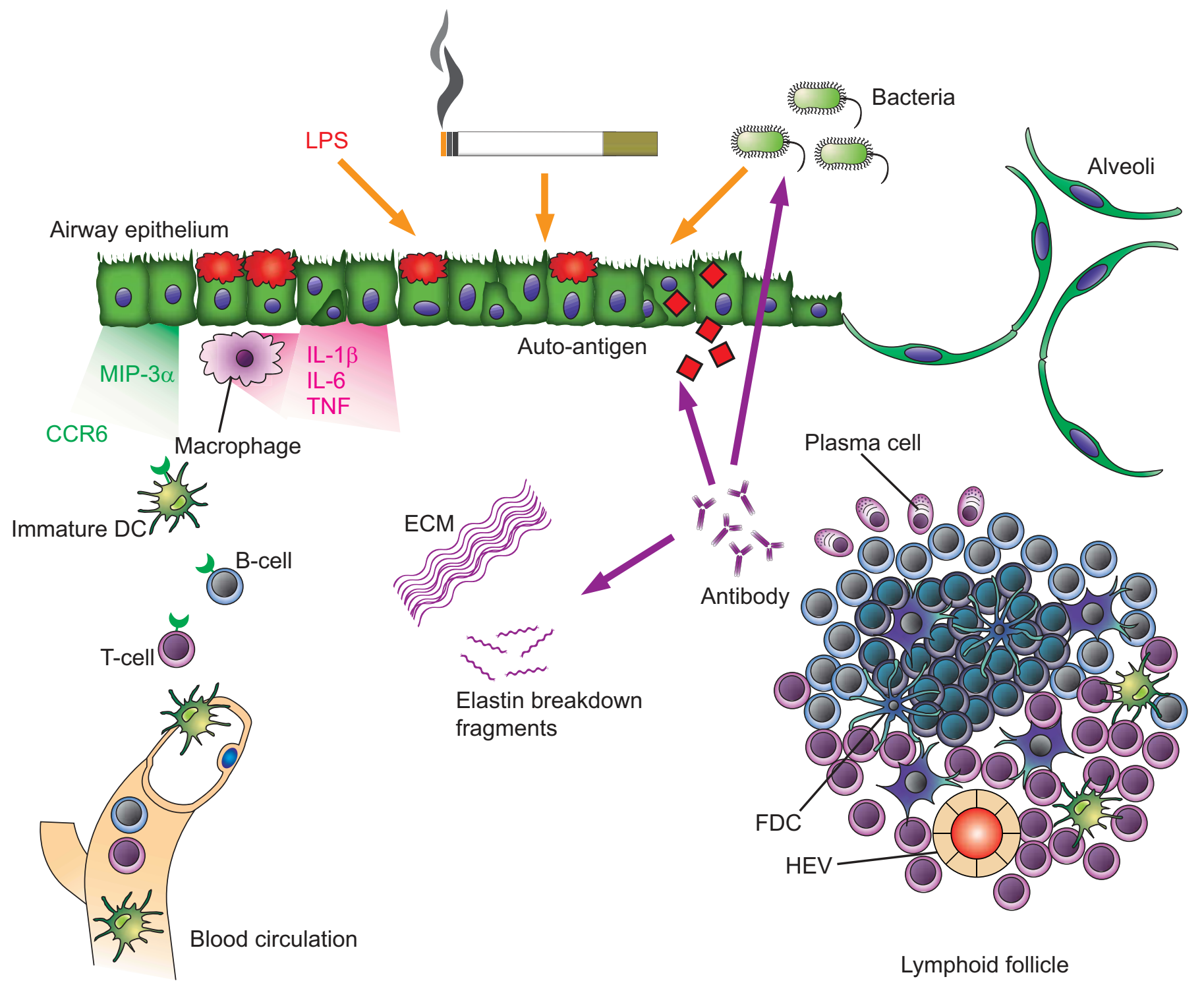

FIGURE 3. Development of lymphoid follicles in chronic obstructive pulmonary disease. In response to environmental insults, such as lipopolysaccharide (LPS), cigarette smoke and bacteria, airway epithelium and macrophages express cytokines (including interleukin (IL)-1 $\beta$, IL-6 and tumour necrosis factor (TNF)- $\alpha$ ) and chemokines (e.g. CCL20/macrophage inflammatory protein (MIP)-3 $\alpha$ ), leading to the accumulation of immature dendritic cells (DCs), T-cells and B-cells. When inflammation becomes chronic due to persisting antigen exposure and/or tissue damage, lymphocyte aggregates will give rise to organised lymphoid follicles with separated B-and T-cell areas. Mature lymphoid follicles may contain high endothelial venules (HEV), follicular dendritic cells (FDC) and germinal centres with the potential to produce plasma cells and antibody responses against bacteria and/or auto-antigens, such as breakdown fragments from the extracellular matrix (ECM). Inhaled and systemic corticosteroids may interfere with several mechanisms involved in the development and function of lymphoid follicles. 


\section{PATHOPHYSIOLOGICAL ROLE OF LYMPHOID FOLLICLES IN COPD}

\section{What are the antigenic stimuli that trigger the formation of lymphoid follicles?}

There are several mechanisms that may underlie the increased numbers of B-cell follicles in lung tissue of COPD patients. The B-cell follicles could reflect, in part, a nonspecific, polyclonal, proliferation of B-cells, induced by local production of B-cell activating mediators including cytokines (IL-4, IL-6, IL-13, TNF- $\alpha$ and lymphotoxin). Alternatively, supported by the demonstration of (oligo)clonality in follicular B-cells in the lung, they might well be the result of a specific humoral immune reaction [11]. At present it is unclear against which antigen(s) this B-cell proliferation could be directed. At least three potential sources of antigens should be considered: 1) microbial; 2) CS components or derivatives; and 3) autoantigens, encompassing epithelial (neo)antigens and degradation products of extracellular matrix.

\section{Chronic bacterial and viral colonisation and/or infection}

The increase in lymphocytes in the small airways and their organisation into bronchial lymphoid follicles are consistent with an adaptive immune response, which is believed to develop in relation to chronic bacterial and/or viral colonisation and infection of the lower airways [44]. Several observations plead in favour of this hypothesis.

First, colonisation of the lower airways by bacteria is a common feature in COPD and, therefore, it is possible that at least part of the reactivity of B- and T-cells in the lymphoid follicles is directed against bacterial antigens [44]. Moreover, microbial colonisation and infection occur more frequently in the later stages of COPD (severe and very severe COPD) than in milder disease; this parallels the increasing frequency of peribronchiolar lymphoid follicles in more severe COPD. Up to $52 \%$ of patients with severe COPD are colonised in the stable state with possible bacterial pathogens, including Haemophilus influenzae, Streptococcus pneumoniae, Moraxella catarrhalis and Pseudomonas aeruginosa [45].

In addition, patients with COPD are prone to frequent exacerbations which are a significant cause of morbidity and mortality. The frequency of COPD exacerbations is modulated by the degree of airway bacterial colonisation in the stable state, and by acquisition of new strains within a pathogenic bacterial species $[44,46,47]$. Using an in vivo model of airway infection in mice, strains of $H$. influenzae isolated from COPD patients during exacerbation induced more airway inflammation than colonisers [48]. Importantly, exacerbation strains are strains which are not only isolated for the first time from sputum of patients experiencing clinical exacerbation symptoms, but are also accompanied by the appearance of new bactericidal antibodies to the homologous infecting strain (compared with serum obtained 1 month before exacerbation). In a cohort study of COPD patients investigating the dynamics of carriage and immune responses to $M$. catarrhalis, MURPHY et al. [49] demonstrated that asymptomatic colonisation was associated with a greater frequency of a sputum IgA response than exacerbation, whereas the intensity of the serum IgG response was greater after exacerbations than colonisation. Since development of new sputum IgA and serum IgG were not correlated, mucosal and systemic immune responses appear to occur independently. Moreover, $M$. catarrhalis was cleared efficiently from the respiratory tract after a short duration of carriage, indicating that COPD patients develop strain-specific protection. In contrast, antibody responses to $P$. aeruginosa developed in $54 \%$ of COPD patients with persistent carriage, suggesting that antibody responses to this bacterium do not mediate clearance, but are a marker for colonisation [50]. Thus, the functional role of the B-cell response to airway bacteria in COPD appears to be strain-dependent.

Secondly, in vivo studies in mice have demonstrated that peribronchial lymphoid aggregates and/or follicles can be induced by live infection with influenza virus or repeated exposure to bacterial lysates of NTHi or bacterial cell wall components (LPS) [31-33]. These animal studies indicate that the chronic exposure of the airways to bacteria and/or bacterial products may be sufficient to induce lymphoid neogenesis. However, VAN DER STRATE et al. [11] elegantly demonstrated that this is not a prerequisite, since the mice that were chronically exposed to CS and developed B-cell follicles were kept under specified pathogen free conditions, together with the air-exposed littermates that did not have these lymphoid follicles. Extensive analysis of fresh frozen samples of the lungs of these mice by RT-PCR did not reveal the presence of microbial species (including Mycoplasma, Chlamydia, adenovirus or Pneumocystis).

\section{Degradation products of extracellular matrix}

Apart from microbial colonisation and infections as the trigger of the development of lymphoid follicles in the lung, an antigen specific immune response against neo- or self antigens present in lung tissue could also be the trigger. In the lungs of COPD patients there is a chronic inflammatory response present with the ongoing recruitment of inflammatory cells and concomitant lung tissue damage. This inflammatory environment can provide the optimal conditions for B-cells to form aggregates and start or sustain a local immune response. This immune response can be directed against lung matrix proteins of the destructed tissue, which can be recognised as neo-antigens. It is known that degradation products of extracellular matrix, such as hyaluronic acid, collagen and elastin, have a chemotactic effect on neutrophils and monocytes [51, 52]. The effects mediated by the inflammatory cells may lead to ongoing degradation of extracellular matrix, which results in maintenance of the chemotactic gradient and therefore inflammation. It is conceivable that B-cells are either locally present or attracted by this gradient, and start producing antibodies against the extracellular matrix degradation products. This is supported by the recent findings of LEE et al. [53], showing the presence of anti-elastin antibodies in plasma and anti-elastin antibody producing B-cells in lung tissue of emphysema patients. The produced antibodies subsequently bind to the fragments of the extracellular matrix, but also to the intact extracellular matrix and cause degradation of extracellular matrix by "frustrated phagocytosis". The follicles in COPD lung tissue resemble the development of similar B-cell follicles in the inflamed synovia in rheumatoid arthritis [54].

The immune response could also be directed against CS components or derivatives which are precipitated in lung tissue, since a low number of these follicles can be observed in "healthy smokers" [13]. CS contains $\sim 4,500$ different compounds [55], of 
which it is likely that several are immunogenic (e.g. tobacco glycoprotein). These compounds will precipitate in the lung, possibly partially bind to the extracellular matrix and may elicit an antibody response. Subsequently, these newly produced antibodies will bind to their antigens, forming an immune complex. Phagocytic immune cells generally remove antigenantibody complexes. If these complexes are either bound to the extracellular matrix, or in the vicinity of it, the extracellular matrix may subsequently be broken down as an "innocent bystander" due to the inflammatory reaction against the immune complexes by these phagocytic cells, similar to rheumatoid arthritis.

Both the mechanisms described for CS- or extracellular matrixderived antigens may also explain the observation that pulmonary inflammation continues in COPD patients after smoking cessation [2-4]. Even though the primary stimulus for inflammation (CS) has vanished, the inflammation continues: it is well known that at least some tobacco residues remain present in the lung for a long time. Direct or indirect antibodymediated degradation of extracellular matrix results in newly formed extracellular matrix-fragments, which may elicit further inflammation.

\section{Hypothesis linking microbial and auto-antigen pathways}

Notwithstanding the notion that the original response of these B-cell follicles in COPD might be protective against bacteria colonising or infecting the lower airways, continued presence of these local immune responses in the lung and the concomitant tissue destruction can promote the breaking of immune tolerance by, for instance, epitope spreading, molecular mimicry or bystander activation (see Appendix). These processes are described to occur after persistent microbial infection or chronic inflammation with the presence of tissue damage. The immune response then extends from the original inducing antigen to similar, often self-reactive epitopes, leading to increased and perpetuated destruction of the inflamed organ and to the initiation of autoimmune responses. In several autoimmune diseases, where similar lymphoid follicles have been shown to be present, these processes have been implicated to play a role in the initiation of the autoimmune response based on findings in animal models. However, there is no direct evidence yet for such an autoimmune mechanism in COPD. Nevertheless, the presence of the chronic inflammatory response in the lungs of COPD patients together with the occurrence of microbial colonisation and recurrent infections, and the presence of lung tissue destruction might provide the circumstances for these processes to occur.

\section{Are lymphoid follicles in COPD protective or harmful?}

Depending on the inducing factors of these lymphoid follicles in the lung, they can be regarded as protective, e.g. protection against infections, or harmful, e.g. by producing autoantibodies and contributing to the perpetuation of the ongoing inflammatory response.

Protection against bacterial and viral colonisation and/or infection Lymphoid neogenesis might have a protective function by inducing a local (and systemic) immune response against the infectious agents. Indirect evidence in favour of a protective role of B-cell follicles in COPD comes from a randomised controlled trial investigating the efficacy and safety of rituximab in patients with COPD. Rituximab is a chimeric human/mouse monoclonal antibody directed against the CD20 antigen, expressed on pre-B-cells and mature B-cells, but not on other cells (including plasma cells). It induces lysis and apoptosis of normal and malignant human B-cells. Rituximab has been long approved for treatment of CD20+ B-cell non-Hodgkin's lymphoma [56, 57], and has recently been approved for treatment of rheumatoid arthritis in patients who have failed TNF-inhibitor therapy $[58,59]$. The study with rituximab in COPD has been terminated early by the data safety monitoring board because of a significant increased risk of infectious complications (including lower respiratory tract infections) in COPD patients treated with rituximab compared with placebo.

\section{Harmful?}

Recently, an increased presence of circulating memory B-cells in peripheral blood of current smokers has been demonstrated, suggesting a smoke-induced specific immune response. Moreover, given the possibility of epitope spreading, molecular mimicry or bystander activation (see Appendix) in COPD, the humoral immune response is likely to be polyreactive and can thus be directed against several epitopes among which are other cellular structures of the inflamed lung. Recently, a high prevalence of anti-Hep-2 epithelial cell autoantibodies in COPD patients was shown, together with a higher presence of antibodies against pulmonary airway epithelial cells in COPD patients compared to healthy controls $[60,61]$. Additionally, TARASEVICIENE-STEWART et al. [62] developed a model of autoimmune emphysema in rats by injection of xenogeneic endothelial cells, and recently showed the presence of anti-endothelial cell antibodies in patients with COPD $[63,64]$. Whether these antibody responses in COPD are pathogenetic and/or true autoimmune responses is still to be determined.

An important characteristic of a pathogenetic autoantibody would be the induction of disease after passive transfer. In the rat model of autoimmune emphysema this was proven by adoptive transfer of the anti-endothelial cell antibodies [62]. For the anti-elastin and anti-epithelial antibodies detected in COPD patients, however, this has not yet been proven and should be addressed in similar animal models. Another characteristic of a pathogenetic autoantibody is the presence in or near the disease specific lesion. This characteristic has not been directly proven in COPD. However, LEE et al. [53] showed the presence of anti-elastin producing B-cells in lung tissue, implying that these antibodies are produced locally in the lung. Additionally, Feghali-Bostwick et al. [60] showed the deposition of IgG immune complexes and complement (C3) in the lungs of COPD patients and demonstrated in vitro that antibodies from COPD patients can exert antibody-mediated cellular cytotoxicity. Both studies are in support of a pathogenetic role for these antibodies in COPD.

\section{INFLUENCE OF PHARMACOTHERAPY ON LYMPHOID FOLLICLES}

Clinical data on the effect of pharmacotherapy on lymphoid follicles are very scarce and indirect, due to the requirement of invasive procedures to obtain relatively large lung samples for 
pathological examination. In the NETT, patients with severe (GOLD 3) and very severe (GOLD 4) COPD were studied longitudinally after lung volume reduction surgery [65]. When the pathological changes of the small airways in the resected lung specimens were (cor)related with clinical outcomes after surgery, the investigators found that occlusion of the small airways by inflammatory exudates containing mucus was associated with early death in these patients with severe emphysema [66]. Intriguingly, there was a strong trend toward a reduction in the number of airways containing lymphoid follicles in COPD patients receiving oral and/or inhaled corticosteroids. The observation that steroid therapy is associated with a reduction in the numbers of follicle-containing airways suggests that steroid-induced suppression of the adaptive immune responses in the peripheral airways might increase the probability of infection in the lower respiratory tract, and may account for the increase in pneumonias observed in recent clinical trials, including TORCH [67] and INSPIRE [68], as well as in observational studies [69]. However, these observations should be interpreted with caution due to the cross-sectional nature of the pathological evaluation in NETT.

We put forward the hypothesis that the increased risk of pneumonias in corticosteroid-treated patients with COPD is partially due to the steroid-induced suppression of adaptive immune responses in general, and of pulmonary B-cell lymphoid follicle formation and function in particular. Several mechanisms might contribute to the suppression of lymphoid follicles by corticosteroids. First, inhaled corticosteroids have profound effects on the airway epithelium, which normally facilitates adaptive immune responses by the synthesis of a wide array of lipid mediators, cytokines and chemokines. Central among the anti-inflammatory properties of corticosteroids is the ability to blunt the infiltration of inflammatory cells, including B- and T-cells, within the airways and lungs through the potent suppression of epithelial-derived cytokines and chemoattractants [70] (fig. 3). Importantly, recent insights into the mechanisms of corticosteroid action in airway epithelium have highlighted a concomitant protective action of corticosteroids on innate immune responses [71, 72] Secondly, corticosteroids also inhibit inflammatory cell migration to sites of inflammation by inhibiting the expression of adhesion molecules on leukocytes and endothelial cells. Thirdly, corticosteroids inhibit epithelial-derived signals (e.g. granulocyte-macrophage colony-stimulating factor and thymic stromal lymphopoietin), which normally enable recruitment, survival and activation of DCs. Upon activation, DCs mature and migrate to regional lymph nodes and presumably also lymphoid follicles, inducing T-cell activation and differentiation (see above) which is modulated by treatment with corticosteroids [73]. Finally, corticosteroids also inhibit T- and B-cell responses directly, by multiple complex mechanisms, including induction of apoptosis in lymphocytes, modulation of B-cell differentiation and inhibition of the expression of effector cytokines (in particular the T-helper 2 cytokines IL-4, IL-5 and IL-13) in T-cells. All these mechanisms may contribute to the tentatively suppressive effects of corticosteroids on the development and function of lymphoid follicles in COPD patients treated with inhaled or systemic corticosteroids (fig. 3).

\section{CONCLUSION AND FUTURE PROSPECTS}

Pathogenesis and progression of severe-to-very severe COPD, in particular, is associated with the presence of peribronchial and parenchymal lymphoid follicles. Using in vivo models of COPD, lymphoid follicles have been described in the lungs of mice which were either chronically exposed to CS or repeatedly exposed to Gram-negative bacteria or LPS. In humans, pulmonary lymphoid neogenesis is not only observed in patients with COPD but also in other chronic diseases of the airways and lungs, including bronchiectasis, follicular bronchiolitis, LIP and pulmonary fibrosis. Outside the lung, lymphoid follicles have been demonstrated in several infectious and autoimmune diseases. Moreover, in some autoimmune diseases, the responsible auto-antigen has been detected within these ectopic lymphoid tissues. The cellular and molecular mechanisms involved in the development of lymphoid follicles are similar to those responsible for the organisation of lymph nodes during organogenesis. The antigenic stimuli that trigger the formation of B-cell follicles in COPD may include microbial antigens, CS components or derivatives, and auto-antigens, encompassing degradation products of extracellular matrix and epithelial (neo)antigens. Epitope spreading, molecular mimicry and bystander activation of B-cells might link the antimicrobial and autoimmune antibody responses observed in patients with severe COPD and emphysema. Therefore, the lymphoid follicles and their ensuing local immune responses might be regarded as either protective (e.g. protection against colonisation and/or infection of the lower respiratory tract) or harmful (e.g. by producing auto-antibodies and perpetuating the ongoing pulmonary inflammation).

We are convinced that the study of the development, structure and function of lymphoid follicles in COPD will provide important clues to a better understanding of the pathogenesis of COPD, including the aetiological agents involved and the mechanisms responsible for the persistent airway inflammation and the relentless progression of the disease. Important questions regarding the role of B-cell follicles and the ensuing antibody responses remain to be addressed. First, since COPD is a heterogeneous disease, encompassing chronic bronchitis, small airways disease (obstructive bronchiolitis) and pulmonary emphysema, does the contribution of lymphoid follicles to the pathogenesis differ between the different compartments they are found in? More research is needed to study the relative contribution of lymphoid follicles in the pathogenesis of the different (e.g. clinical, radiological, physiological and pathological) phenotypes of COPD [74-76]. Secondly, does the presence of auto-antibodies implicate that COPD is really an autoimmune disease, where B-cell responses drive the pathogenetic mechanisms, or are these auto-antibodies only an epiphenomenon occurring late in the course of the disease (i.e. in end-stage COPD) and, thus, should they be regarded as innocent bystanders? Are bacterial colonisation and/or infection of the lower respiratory tract a prerequisite for the development of lymphoid follicles in patients with COPD? Is there any link between the systemic manifestations of COPD and the development of lymphoid follicles? What is the effect of current and future pharmacotherapy for COPD on lymphoid follicles? Finally and most importantly, are pulmonary B-cell follicles and responses a worthwhile target in the treatment of (specific subgroups of) COPD patients? 


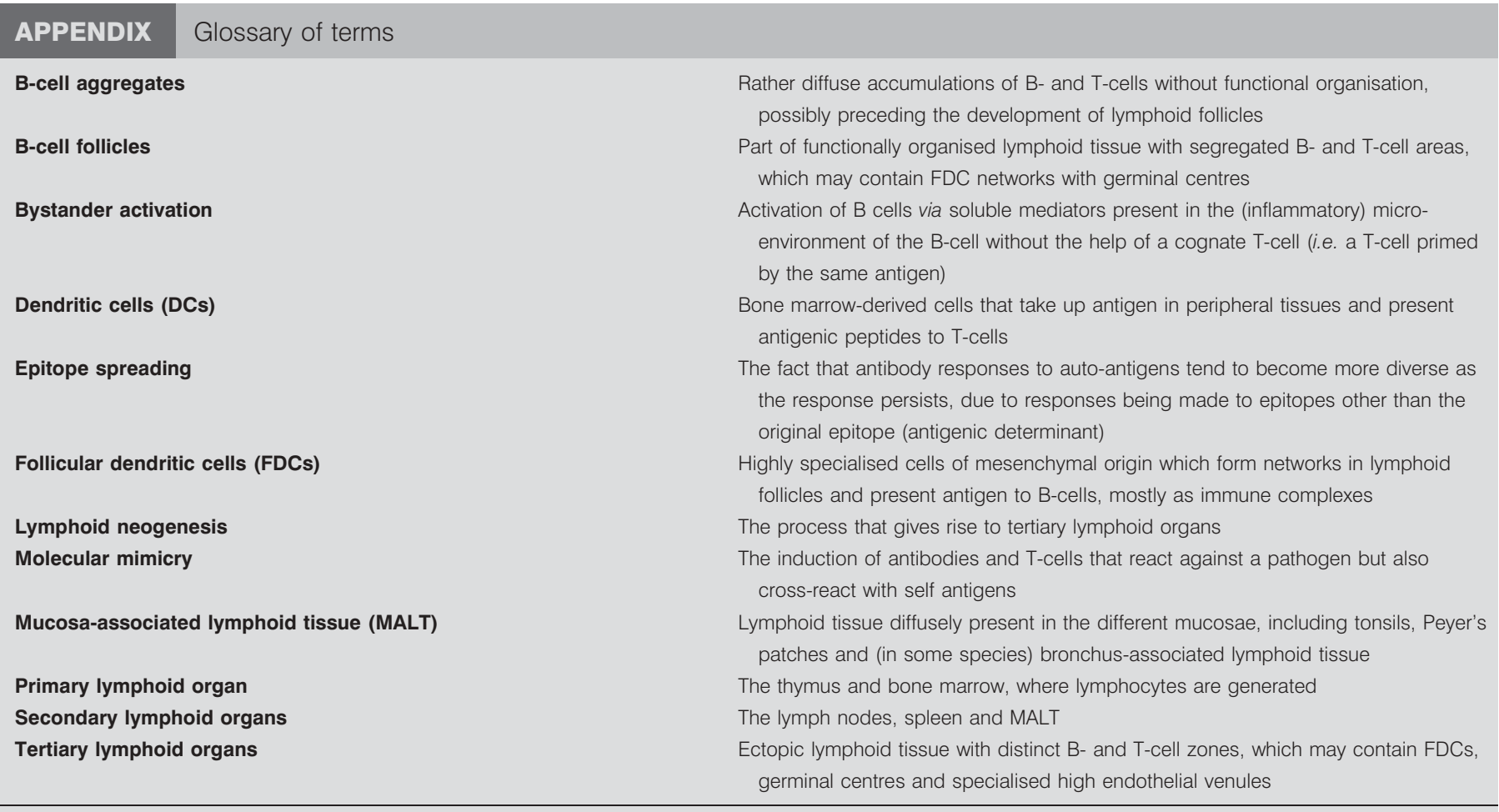

\section{SUPPORT STATEMENT}

This work was supported by the Concerted Research Action of the University of Ghent (Ghent, Belgium; BOF/GOA 01251504) and by the Netherlands Asthma Fund. K.R. Bracke is a postdoctoral fellow sponsored by the Fonds voor Wetenschappelijk OnderzoekVlaanderen (Brussels, Belgium).

\section{STATEMENT OF INTEREST}

A statement of interest for G.G. Brusselle can be found at www.erj. ersjournals.com $/ \mathrm{misc} /$ statements.dtl

\section{REFERENCES}

1 Pauwels RA, Rabe KF. Burden and clinical features of chronic obstructive pulmonary disease (COPD). Lancet 2004; 364: 613-620.

2 Rutgers SR, Postma DS, ten Hacken NH, et al. Ongoing airway inflammation in patients with COPD who do not currently smoke. Thorax 2000; 55: 12-18.

3 Willemse BW, ten Hacken NH, Rutgers B, et al. Effect of 1-year smoking cessation on airway inflammation in COPD and asymptomatic smokers. Eur Respir J 2005; 26: 835-845.

4 Gamble E, Grootendorst DC, Hattotuwa K, et al. Airway mucosal inflammation in COPD is similar in smokers and ex-smokers: a pooled analysis. Eur Respir J 2007; 30: 467-471.

5 Pauwels RA, Buist AS, Calverley PM, et al. Global strategy for the diagnosis, management, and prevention of chronic obstructive pulmonary disease. NHLBI/WHO Global Initiative for Chronic Obstructive Lung Disease (GOLD) Workshop summary. Am J Respir Crit Care Med 2001; 163: 1256-1276.

6 Hautamaki RD, Kobayashi DK, Senior RM, et al. Requirement for macrophage elastase for cigarette smoke-induced emphysema in mice. Science 1997; 277: 2002-2004.

7 D'hulst AI, Vermaelen KY, Brusselle GG, et al. Time course of cigarette smoke-induced pulmonary inflammation in mice. Eur Respir J 2005; 26: 204-213.
8 Saetta M, Di SA, Turato G, et al. CD8+ T-lymphocytes in peripheral airways of smokers with chronic obstructive pulmonary disease. Am J Respir Crit Care Med 1998; 157: 822-826.

9 Cosio MG, Guerassimov A. Chronic obstructive pulmonary disease. Inflammation of small airways and lung parenchyma. Am J Respir Crit Care Med 1999; 160: S21-S25.

10 Hogg JC, Chu F, Utokaparch S, et al. The nature of small-airway obstruction in chronic obstructive pulmonary disease. $N$ Engl J Med 2004; 350: 2645-2653.

11 van der Strate BW, Postma DS, Brandsma CA, et al. Cigarette smoke-induced emphysema: a role for the B cell? Am J Respir Crit Care Med 2006; 173: 751-758.

12 Gosman MM, Willemse BW, Jansen DF, et al. Increased number of B-cells in bronchial biopsies in COPD. Eur Respir J 2006; 27: 60-64.

13 Bosken $\mathrm{CH}$, Hards J, Gatter $\mathrm{K}$, et al. Characterization of the inflammatory reaction in the peripheral airways of cigarette smokers using immunocytochemistry. Am Rev Respir Dis 1992; 145: 911-917.

14 Hogg JC. Pathophysiology of airflow limitation in chronic obstructive pulmonary disease. Lancet 2004; 364: 709-721.

15 Elliot JG, Jensen CM, Mutavdzic S, et al. Aggregations of lymphoid cells in the airways of nonsmokers, smokers, and subjects with asthma. Am J Respir Crit Care Med 2004; 169: 712-718.

16 Aloisi F, Pujol-Borrell R. Lymphoid neogenesis in chronic inflammatory diseases. Nat Rev Immunol 2006; 6: 205-217.

17 Kroese FGM, Timens W, Nieuwenhuis P. Germinal center reaction and B lymphocytes: morphology and function. Curr Top Pathol 1990; 84: 103-148.

18 MacLennan IC. Germinal centers. Annu Rev Immunol 1994; 12 : 117-139.

19 Zubler RH. Naïve and memory B cells in T-cell-dependent and Tindependent responses. Springer Semin Immunopathol 2001; 23: 405419.

20 Pabst R, Gehrke I. Is the bronchus-associated lymphoid tissue (BALT) an integral structure of the lung in normal mammals, including humans? Am J Respir Cell Mol Biol 1990; 3: 131-135. 
21 Carragher DM, Rangel-Moreno J, Randall TD. Ectopic lymphoid tissues and local immunity. Semin Immunol 2008; 20: 26-42.

22 Hjelmstrom P. Lymphoid neogenesis: de novo formation of lymphoid tissue in chronic inflammation through expression of homing chemokines. J Leukoc Biol 2001; 69: 331-339.

23 Drayton DL, Liao S, Mounzer RH, et al. Lymphoid organ development: from ontogeny to neogenesis. Nat Immunol 2006; 7 344-353.

24 Brusselle GG, Bracke KR, Maes T, et al. Murine models of COPD. Pulm Pharmacol Ther 2006; 19: 155-165.

25 Shapiro SD. Transgenic and gene-targeted mice as models for chronic obstructive pulmonary disease. Eur Respir J 2007; 29: 375-378.

26 Churg A, Cosio M, Wright JL. Mechanisms of cigarette smokeinduced COPD: insights from animal models. Am J Physiol Lung Cell Mol Physiol 2008; 294: L612-L631.

27 D'hulst AI, Maes T, Bracke KR, et al. Cigarette smoke-induced pulmonary emphysema in scid-mice. Is the acquired immune system required? Respir Res 2005; 6: 147.

28 Bracke KR, D'hulst AI, Maes T, et al. Cigarette smoke-induced pulmonary inflammation and emphysema are attenuated in CCR6-deficient mice. I Immunol 2006; 177: 4350-4359.

29 Maes T, Bracke KR, Vermaelen KY, et al. Murine TLR4 is implicated in cigarette smoke-induced pulmonary inflammation. Int Arch Allergy Immunol 2006; 141: 354-368.

30 Bracke KR, D'hulst AI, Maes T, et al. Cigarette smoke-induced pulmonary inflammation, but not airway remodelling, is attenuated in chemokine receptor 5-deficient mice. Clin Exp Allergy 2007, 37: 1467-1479.

31 Moghaddam SJ, Clement CG, De la Garza MM, et al. Haemophilus influenzae lysate induces aspects of the chronic obstructive pulmonary disease phenotype. Am J Respir Cell Mol Biol 2008; 38: 629-638

32 Vernooy JH, Dentener MA, van Suylen RJ, et al. Long-term intratracheal lipopolysaccharide exposure in mice results in chronic lung inflammation and persistent pathology. Am J Respir Cell Mol Biol 2002; 26: 152-159.

33 Moyron-Quiroz JE, Rangel-Moreno J, Kusser K, et al. Role of inducible bronchus associated lymphoid tissue (iBALT) in respiratory immunity. Nat Med 2004; 10: 927-934.

34 Lappalainen U, Whitsett JA, Wert SE, et al. Interleukin-1 $\beta$ causes pulmonary inflammation, emphysema, and airway remodeling in the adult murine lung. Am J Respir Cell Mol Biol 2005; 32: 311-318.

35 Goya S, Matsuoka H, Mori M, et al. Sustained interleukin-6 signalling leads to the development of lymphoid organ-like structures in the lung. J Pathol 2003; 200: 82-87.

36 Miyazaki Y, Araki K, Vesin C, et al. Expression of a tumor necrosis factor-alpha transgene in murine lung causes lymphocytic and fibrosing alveolitis. A mouse model of progressive pulmonary fibrosis. J Clin Invest 1995; 96: 250-259.

37 Fujita M, Shannon JM, Irvin CG, et al. Overexpression of tumor necrosis factor-alpha produces an increase in lung volumes and pulmonary hypertension. Am J Physiol Lung Cell Mol Physiol 2001; 280: L39-L49.

38 Lundblad LK, Thompson-Figueroa J, Leclair T, et al. Tumor necrosis factor-alpha overexpression in lung disease: a single cause behind a complex phenotype. Am J Respir Crit Care Med 2005; 171: 1363-1370.

39 De Togni P, Goellner J, Ruddle NH, et al. Abnormal development of peripheral lymphoid organs in mice deficient in lymphotoxin. Science 1994; 264: 703-707.

40 Futterer A, Mink K, Luz A, et al. The lymphotoxin beta receptor controls organogenesis and affinity maturation in peripheral lymphoid tissues. Immunity 1998; 9: 59-70.

41 Mebius RE. Organogenesis of lymphoid tissues. Nat Rev Immunol 2003; 3: 292-303

42 Vermaelen K, Pauwels R. Pulmonary dendritic cells. Am J Respir Crit Care Med 2005; 172: 530-551.
43 Demedts IK, Bracke KR, Van PG, et al. Accumulation of dendritic cells and increased CCL20 levels in the airways of patients with chronic obstructive pulmonary disease. Am J Respir Crit Care Med 2007; 175: 998-1005.

44 Sethi S, Murphy TF. Infection in the pathogenesis and course of chronic obstructive pulmonary disease. $N$ Engl J Med 2008; 359: 2355-2365.

45 Patel IS, Seemungal TA, Wilks M, et al. Relationship between bacterial colonisation and the frequency, character, and severity of COPD exacerbations. Thorax 2002; 57: 759-764.

46 Sethi S, Evans N, Grant BJ, et al. New strains of bacteria and exacerbations of chronic obstructive pulmonary disease. $N$ Engl J Med 2002; 347: 465-471.

47 Sethi S, Sethi R, Eschberger K, et al. Airway bacterial concentrations and exacerbations of chronic obstructive pulmonary disease. Am J Respir Crit Care Med 2007; 176: 356-361.

48 Chin CL, Manzel LJ, Lehman EE, et al. Haemophilus influenzae from patients with chronic obstructive pulmonary disease exacerbation induce more inflammation than colonizers. Am J Respir Crit Care Med 2005; 172: 85-91.

49 Murphy TF, Brauer AL, Grant BJ, et al. Moraxella catarrhalis in chronic obstructive pulmonary disease: burden of disease and immune response. Am J Respir Crit Care Med 2005; 172: 195-199.

50 Murphy TF, Brauer AL, Eschberger K, et al. Pseudomonas aeruginosa in chronic obstructive pulmonary disease. Am J Respir Crit Care Med 2008; 177: 853-860.

51 McKee CM, Penno MB, Cowman M, et al. Hyaluronan (HA) fragments induce chemokine gene expression in alveolar macrophages. The role of HA size and CD44. J Clin Invest 1996; 98: 2403-2413.

52 Horton MR, Shapiro S, Bao C, et al. Induction and regulation of macrophage metalloelastase by hyaluronan fragments in mouse macrophages. J Immunol 1999; 162: 4171-4176.

53 Lee SH, Goswami S, Grudo A, et al. Antielastin autoimmunity in tobacco smoking-induced emphysema. Nat Med 2007; 13: 567-569.

54 Magalhaes R, Stiehl P, Morawietz L, et al. Morphological and molecular pathology of the $B$ cell response in synovitis of rheumatoid arthritis. Virchows Arch 2002; 441: 415-427.

55 Sopori M. Effects of cigarette smoke on the immune system. Nat Rev Immunol 2002; 2: 372-377.

56 Cvetkovic RS, Perry CM. Rituximab: a review of its use in nonHodgkin's lymphoma and chronic lymphocytic leukaemia. Drugs 2006; 66: 791-820.

57 Cheson BD, Leonard JP. Monoclonal antibody therapy for B-cell non-Hodgkin's lymphoma. N Engl J Med 2008; 359: 613-626.

58 Browning JL. B cells move to centre stage: novel opportunities for autoimmune disease treatment. Nat Rev Drug Discov 2006; 5 564-576.

59 Smolen JS, Aletaha D, Koeller M, et al. New therapies for treatment of rheumatoid arthritis. Lancet 2007; 370: 1861-1874.

60 Feghali-Bostwick CA, Gadgil AS, Otterbein LE, et al. Autoantibodies in patients with chronic obstructive pulmonary disease. Am J Respir Crit Care Med 2008; 177: 156-163.

61 Bonarius HP, Timens W, Brandsma CA, et al. Elevated antiepithelial autoantibodies titer and increased cationic amino acids in IgH-CDR3 in chronic obstructive pulmonary disease. Am J Respir Crit Care Med 2008; 177: A657.

62 Taraseviciene-Stewart L, Scerbavicius R, Choe KH, et al. An anima model of autoimmune emphysema. Am J Respir Crit Care Med 2005 171: 734-742.

63 Taraseviciene-Stewart L, Douglas IS, Nana-Sinkam PS, et al. Is alveolar destruction and emphysema in chronic obstructive pulmonary disease an immune disease? Proc Am Thorac Soc 2006; 3: 687-690.

64 Taraseviciene-Stewart L, Voelkel NF. Molecular pathogenesis of emphysema. J Clin Invest 2008; 118: 394-402. 
65 Fishman A, Martinez F, Naunheim K, et al. A randomized trial comparing lung-volume-reduction surgery with medical therapy for severe emphysema. N Engl J Med 2003; 348: 2059-2073.

66 Hogg JC, Chu FS, Tan WC, et al. Survival after lung volume reduction in chronic obstructive pulmonary disease: insights from small airway pathology. Am J Respir Crit Care Med 2007; 176: 454-459.

67 Calverley PM, Anderson JA, Celli B, et al. Salmeterol and fluticasone propionate and survival in chronic obstructive pulmonary disease. N Engl J Med 2007; 356: 775-789.

68 Wedzicha JA, Calverley PM, Seemungal TA, et al. The prevention of chronic obstructive pulmonary disease exacerbations by salmeterol/fluticasone propionate or tiotropium bromide. Am J Respir Crit Care Med 2008; 177: 19-26.

69 Ernst P, Gonzalez AV, Brassard P, et al. Inhaled corticosteroid use in chronic obstructive pulmonary disease and the risk of hospitalization for pneumonia. Am J Respir Crit Care Med 2007; 176: 162-166.

70 Stellato C. Glucocorticoid actions on airway epithelial responses in immunity: functional outcomes and molecular targets. J Allergy Clin Immunol 2007; 120: 1247-1263.
71 Schleimer RP. Glucocorticoids suppress inflammation but spare innate immune responses in airway epithelium. Proc Am Thorac Soc 2004; 1: 222-230.

72 Zhang N, Truong-Tran QA, Tancowny B, et al. Glucocorticoids enhance or spare innate immunity: effects in airway epithelium are mediated by CCAAT/enhancer binding proteins. J Immunol 2007; 179: 578-589.

73 Georas SN. Inhaled glucocorticoids, lymphocytes, and dendritic cells in asthma and obstructive lung diseases. Proc Am Thorac Soc 2004; 1: 215-221.

74 Dransfield MT, Washko GR, Foreman MG, et al. Gender differences in the severity of CT emphysema in COPD. Chest 2007; 132: 464-470.

75 Makita H, Nasuhara Y, Nagai K, et al. Characterisation of phenotypes based on severity of emphysema in chronic obstructive pulmonary disease. Thorax 2007; 62: 932-937.

76 Omori H, Fujimoto K, Katoh T. Computed-tomography findings of emphysema: correlation with spirometric values. Curr Opin Pulm Med 2008; 14: 110-114. 